\title{
Using Determining Sets to Distinguish Kneser Graphs
}

\author{
Michael O. Albertson \\ Department of Mathematics and Statistics \\ Smith College, Northampton MA 01063 \\ albertson@math.smith.edu
}

\author{
Debra L. Boutin \\ Department of Mathematics \\ Hamilton College, Clinton, NY 13323 \\ dboutin@hamilton.edu
}

Submitted: Sep 10, 2006; Accepted: Jan 19, 2007; Published: Jan 29, 2007

Mathematics Subject Classification: 05C25, 05C78

\begin{abstract}
This work introduces the technique of using a carefully chosen determining set to prove the existence of a distinguishing labeling using few labels. A graph $G$ is said to be $d$-distinguishable if there is a labeling of the vertex set using $1, \ldots, d$ so that no nontrivial automorphism of $G$ preserves the labels. A set of vertices $S \subseteq V(G)$ is a determining set for $G$ if every automorphism of $G$ is uniquely determined by its action on $S$. We prove that a graph is $d$-distinguishable if and only if it has a determining set that can be $(d-1)$-distinguished. We use this to prove that every Kneser graph $K_{n: k}$ with $n \geq 6$ and $k \geq 2$ is 2-distinguishable.
\end{abstract}

\section{Introduction}

The distinguishing number of a graph $G$ is the smallest integer $d$ so that each vertex of $G$ can be labeled with an integer from $\{1, \ldots, d\}$ in such a way that no automorphism of $G$, other than the identity, preserves the labels. Albertson and Collins introduced distinguishing in [3]. There has been a flurry of activity on distinguishing in the last few years: see e.g. $[1,2,4,6,7,8,9,13,14,15,17,18,20]$.

A subset $S$ of vertices of a graph $G$ is called a determining set if whenever two automorphisms agree on the elements of $S$, they agree on all of $G$. That is, the image of $S$ under an arbitrary automorphism determines the automorphism completely. The determining number of a graph $G$ is the smallest integer $r$ so that $G$ has a determining set of size $r$. Boutin introduced determining in [5].

Both distinguishing labelings and determining sets illuminate and quantify the symmetry of a graph. However, there are fundamental differences between these two concepts. A distinguishing labeling adds a new aspect (vertex labels) to the graph in order to break all graph symmetries. In this situation labeling two vertices with different labels may effectively 'break' more than one automorphism. In contrast a determining set, while 
adding nothing new to the graph, seeks to capture the differences between automorphisms. If multiple automorphisms have the same action on a given pair of vertices, those two vertices alone are not sufficient to differentiate the automorphisms in question. More information (in the form of more vertices in the determining set) is needed.

We see in Theorem 3 that if a graph has determining number $k$, its distinguishing number is at most $k+1$. However, a graph may have small distinguishing number and large determining number. For example, the determining number of the Kneser graph $K_{n: k}$ is at least $\log _{2}(n+1)$ [5], while we see in Theorem 4 that if $n \geq 6$ and $k \geq 2$, its distinguishing number is only 2 . Thus the difference between the distinguishing number and the determining number for a given graph can be arbitrarily large.

Determining sets provide a useful tool for finding distinguishing numbers. Theorem 3 tells us that to prove $G$ has distinguishing number at most $k+1$, it suffices to find a determining set that can be $k$-distinguished. In particular, to prove that $\mathrm{G}$ has distinguishing number at most 2, it suffices to find (a superset of) a determining set that can be 1-distinguished (as a subset) or whose induced subgraph is asymmetric. We use this method to find the distinguishing numbers for Kneser graphs.

The paper is organized as follows. Section 2 gives background on both distinguishing numbers and determining numbers. The connections between distinguishing and determining are exposed in Section 3. Finally, Section 4 establishes the distinguishing numbers of the Kneser graphs.

\section{Background}

Definition 1. A labeling $f: V(G) \rightarrow\{1, \ldots, d\}$ is said to be $d$-distinguishing if $\phi \in$ $\operatorname{Aut}(G)$ and $f(\phi(x))=f(x)$ for all $x \in V(G)$ implies that $\phi=i d$. The distinguishing number of $G$, denoted here by $\operatorname{Dist}(G)$, is the minimum $d$ such that $G$ has a $d$-distinguishing labeling.

Note that we have a $d$-distinguishing labeling of a graph $G$ if and only if the labeling together with the structure of $G$ uniquely identifies every vertex. Every graph has a distinguishing labeling since we can label each vertex with a different integer from $\{1, \cdots,|V(G)|\}$. Furthermore, there are graphs, e.g. $K_{n}$ and $K_{1, n}$, for which such a labeling is optimal or nearly optimal. Also note that $\operatorname{Dist}(G)=\operatorname{Dist}\left(G^{c}\right)$.

The distinguishing number, introduced in [3], was inspired by a recreational puzzle of Frank Rubin [16]. Rubin's puzzle is (in the current language) to find $\operatorname{Dist}\left(C_{n}\right)$. One attraction of the puzzle is its contrasting solutions. While $\operatorname{Dist}\left(C_{3}\right)=\operatorname{Dist}\left(C_{4}\right)=\operatorname{Dist}\left(C_{5}\right)=3$, when $n \geq 6$, $\operatorname{Dist}\left(C_{n}\right)=2$.

Definition 2. A subset $S \subseteq V(G)$ is said to be a determining set if whenever $g, h \in$ Aut $(G)$ and $g(x)=h(x)$ for all $x \in S$, then $g=h$. The determining number of $G$, denoted here by $\operatorname{Det}(G)$, is the minimum $r$ such that $G$ has a determining set of cardinality $r$ [5]. 
Another way to think about determining sets is that $S$ is a determining set if and only if every vertex in the graph can be uniquely identified by its relationship to the vertices in $S$. A basis for a vector space is an analogue of a determining set. Thus in a vector space the determining number is just the dimension. An early form of a determining set is the concept of a base for a group action. Formally, a base for a permutation action on a set $\Omega$ is a subset of $\Omega$ such that the only permutation of $\Omega$ that fixes every element in the subset is the identity. [11]

Every graph has a determining set, since any set containing all but one vertex is determining. There are graphs, e.g. $K_{n}$ and $K_{1, n}$, for which such a determining set is optimal or nearly optimal. Also note that $\operatorname{Det}(G)=\operatorname{Det}\left(G^{c}\right)$.

Every $n$-cycle has a determining set consisting of any two non-antipodal vertices. Note that for $n \geq 6$, $\operatorname{Dist}\left(C_{n}\right)=\operatorname{Det}\left(C_{n}\right)=2$, but for $3 \leq n \leq 5$, $\operatorname{Dist}\left(C_{n}\right)=3$ while $\operatorname{Det}\left(C_{n}\right)=2$.

Recall that $\operatorname{Stab}(S)=\cap_{s \in S} \operatorname{Stab}(s)$ is the pointwise stabilizer of the set $S$. The following theorem characterizes determining sets in terms of stabilizers and will be useful in the next section. Its proof is straightforward.

Theorem 1. [5] Let $S$ be a subset of the vertices of the graph $G$. Then $S$ is a determining set for $G$ if and only if $\operatorname{Stab}(S)=\{i d\}$.

\section{Linking Distinguishing and Determining}

We already know what it means for (the set of vertices of) a graph to be $d$-distinguished. In order to connect determining sets and distinguishing labelings we also need to know what it means for a subset of vertices to be $d$-distinguished.

Definition 3. Let $S \subseteq V(G)$. A labeling $f: S \rightarrow\{1, \cdots, d\}$ is called d-distinguishing if $\phi \in \operatorname{Aut}(G)$ and $f(\phi(x))=f(x)$ for all $x \in S$ implies that $\phi \in \operatorname{Stab}(S)$.

That is, $\phi$ may not be the identity (it might move vertices that are not in $S$ ), but $\phi$ fixes $S$ pointwise.

Theorem 2. Let $S \subseteq V(G)$. Let $H$ be the subgraph of $G$ induced by $S$. A $d$ distinguishing labeling for $H$ as a graph induces a $d$-distinguishing labeling for $S$ as a subset of $V(G)$.

Proof. Find a $d$-distinguishing labeling for $H$ and use this same labeling for the vertices of $S$. Leave the remaining vertices of $G$ unlabeled. Suppose that $\phi \in \operatorname{Aut}(G)$ preserves labels (and non-labels). Since $\phi$ preserves the property of being labeled it induces a bijection on $S$. Further the induced bijection preserves labels, adjacency, and non-adjacency among the vertices of $S$. Thus $\phi$ induces a label preserving automorphism of $H$, which implies that $\phi$ induces the identity automorphism on $H$. That is, as a permutation $\phi$ fixes the vertices of $H$; but these are the vertices of $S$. Thus $\phi \in \operatorname{Stab}(S)$. 
Note that it may be possible to distinguish a subset of vertices in a graph with fewer labels than are needed to distinguish its induced subgraph. For example, consider $C_{6}$. Let $S$ consist of two adjacent vertices and a third vertex not adjacent to either of the other two. The subgraph induced by this set is an edge and an isolated vertex. Its distinguishing number is 2. However, when viewed as a subset of $C_{6}, S$ is 1-distinguishable. Any automorphism that flips the edge must transpose the third vertex with a vertex outside the set, which has no label. This is impossible.

Theorem 3. $G$ has a $d$-distinguishable determining set if and only if $G$ can be $(d+1)$ distinguished.

Proof. If $G$ has a $d$-distinguishable determining set $S$, then so distinguish it. Label all vertices not in $S$ with the label $d+1$. If $\phi \in \operatorname{Aut}(G)$ preserves labels of $G$, then it preserves the labels of $S$. Because this is a distinguishing labeling for $S, \phi \in \operatorname{Stab}(S)$. Since $S$ is a determining set, $\operatorname{Stab}(S)=\{i d\}$. Thus $\phi=i d$ and the labeling of $G$ is $(d+1)$-distinguishing.

Suppose that $G$ is $(d+1)$-distinguishable. Then so distinguish it. Let $S$ be the set of vertices with labels $1, \cdots, d$. We want to show that $S$ is both $d$-distinguished and a determining set. Suppose that $\phi \in \operatorname{Aut}(G)$ preserves the given labels of $S$. Then it preserves the labels of the remaining vertices of $G$. But since this is a distinguishing labeling of $G, \phi=i d$, which implies that $\phi \in \operatorname{Stab}(S)$. Thus we have a $d$-distinguishing labeling for $S$. Now suppose that $\phi \in \operatorname{Stab}(S)$. Then it preserves labels $1, \cdots, d$. Furthermore, since vertices outside of $S$ can only be sent to vertices outside of $S, \phi$ also preserves the label $d+1$. Thus $\phi$ preserves all labels which implies that $\phi=i d$. Thus $\operatorname{Stab}(S)=\{i d\}$ and therefore $S$ is a determining set.

Note that by the theorems above, if we can find a determining set for $G$ so that the subgraph induced by it is $d$-distinguishable, then $G$ is $(d+1)$-distinguishable. We'll use this with $d=1$ in the following section.

\section{Distinguishing Kneser Graphs}

Let $K_{n: k}$ denote the Kneser graph where $n \geq 2 k+1$. Here $V\left(K_{n: k}\right)$ consists of all of the $\left(\begin{array}{l}n \\ k\end{array}\right) k$-element subsets of $[n]=\{1,2, \ldots n\}$, and $u v \in E\left(K_{n: k}\right)$ when the subsets corresponding to $u$ and $v$ have empty intersection. It is well known that automorphisms of $K_{n: k}$ correspond to permutations of $[n]$; thus $\operatorname{Aut}\left(K_{n: k}\right) \cong S_{n}$ [10]. It is immediate that $K_{n: 1} \cong K_{n}$ : thus $\operatorname{Dist}\left(K_{n: 1}\right)=n$. The Petersen graph is $K_{5: 2}$, and it is known that $\operatorname{Dist}\left(K_{5: 2}\right)=3[3]$. Our purpose in this section is to show that these are the only Kneser graphs that are not 2-distinguishable.

Theorem 4. If $k \geq 2$ and $n \neq 5$, then $\operatorname{Dist}\left(K_{n: k}\right)=2$. 
Proof. It is straightforward to see that $K_{n: 2}$ is isomorphic to $\left(L\left(K_{n}\right)\right)^{c}$, the complement of the line graph of the $n$-clique. Lovasz observed that when $n \geq 6$, $\operatorname{Dist}\left(\left(L\left(K_{n}\right)\right)^{c}\right)=2$ [3]. Thus we may assume that $k \geq 3$.

Throughout this proof all addition will be modulo $n$. Furthermore, when we say that two integers are consecutive, that also means modulo $n$. Also, we use $n$ rather than 0 to denote integers that are evenly divisible by $n$.

We wish to give a special notation to a subset of the vertices of $K_{n: k}$. Let $V_{i}$ denote the vertex that corresponds to the $k$-element subset $\{i, i+1, \ldots, i+k-1\}$. Notice that $V_{i} V_{j} \in E\left(K_{n: k}\right)$ when $k \leq|j-i| \leq n-k$. Boutin has shown that $\left\{V_{1}, V_{2}, \ldots, V_{n-k}\right\}$ is a determining set for $K_{n: k}[5]$. In each of the two following constructions we find a superset of this determining set that induces a 1-distinguishable subgraph. Using Theorems 2 and 3 then yield our result.

Case 1) Suppose that $n \geq 3 k$. In this case we prove that the complement of $K_{n: k}$ is 2distinguishable, which proves that $K_{n: k}$ itself is 2-distinguishable. Note that in $\left(K_{n: k}\right)^{c}$ two vertices are adjacent precisely when their associated subsets have nontrivial intersection. Let $F$ denote the subgraph of $\left(K_{n: k}\right)^{c}$ induced by $\left\{V_{i}: 1 \leq i \leq n-k+1\right\}$. Since any superset of a determining set is determining, $V(F)$ is also a determining set. It is worth remarking that $F$ is just a power of a path. Specifically $F \cong P_{n-k+1}^{k-1}$ where $P_{n-k+1}$ is the path with $n-k+1$ vertices. (That is, two vertices in $F$ are adjacent if their distance in $P_{n-k+1}$ is at most $k-1$.) Note that within $F$, vertex $V_{j}$ is adjacent to $\min \{k-1, j-1\}$ vertices to its 'left' and $\min \{k-1, n-k+1-j\}$ vertices to its 'right'. Thus $\operatorname{deg}_{F}\left(V_{j}\right)=k+j-2$ if $1 \leq j \leq k-1, \operatorname{deg}_{F}\left(V_{j}\right)=2 k-2$ if $k \leq j \leq n-2 k+2$ and $\operatorname{deg}_{F}\left(V_{j}\right)=k-1+(n-k+1-j)=n-j$ if $n-2 k+3 \leq j \leq n-k+1$.

We now add one additional vertex to $V(F)$ to create an asymmetric induced subgraph of $\left(K_{n: k}\right)^{c}$. Let $Y$ denote the vertex that corresponds to the subset $\{2,4, \ldots, 2 k\}$. Let $G=$ $G_{n: k}$ denote the subgraph of $\left(K_{n: k}\right)^{c}$ induced by $\left\{Y, V_{1}, \ldots, V_{n-k+1}\right\}$. Figure 1 shows $G_{10: 3}$. It is immediate that $Y$ is adjacent (within $G$ ) to precisely $V_{1}, \ldots, V_{2 k}$. Using the degrees we computed above for $F$, it is straightforward to see that $\operatorname{deg}_{G}(Y)=2 k$, $\operatorname{deg}_{G}\left(V_{j}\right)=k-1+j$ for $1 \leq j \leq k-1$ and $\operatorname{deg}_{G}\left(V_{j}\right)=2 k-1$ for $k \leq j \leq \min \{2 k, n-2 k+2\}$. Further, since $n \geq 3 k, n-k+1 \geq 2 k+1$ which implies that $Y$ is not adjacent to $V_{n-k+1}$. Thus $\operatorname{deg}_{G}\left(V_{n-k+1}\right)=n-(n-k+1)=k-1$. (We won't specifically need the degree when $\min \{2 k, n-2 k+2\}<j<n-k+1$.)

We now show that $G$ is 1-distinguishable. Any automorphism of $G$ must fix $Y$ and $V_{n-k+1}$, since these are the unique vertices of maximum and minimum degrees. Suppose $\sigma \in \operatorname{Aut}(G)$. Then since $\sigma$ fixes $Y$, it induces a permutation, $\hat{\sigma}$, on the vertices of $F$. But $\hat{\sigma}$ must preserve adjacency among the vertices of $F$ since it preserves their adjacencies as vertices of $G$. Thus $\hat{\sigma} \in \operatorname{Aut}(F)$. Thus any automorphism of $G$ is an automorphism of $F$ that fixes $V_{n-k+1}$. If $V_{n-k+1}$ is fixed, then so is $V_{1}$, since these are the two vertices in $F$ whose degrees equal $k-1$. For $2 \leq j \leq k-1$ the vertex $V_{j}$ is adjacent to $V_{1}$ and $\operatorname{deg}_{F}\left(V_{j}\right)=k-2+j$. These two properties uniquely identify each such $V_{j}$. For $k \leq j \leq n-2 k+2, \operatorname{deg}_{F}\left(V_{j}\right)=2 k-2$. For each such $j, V_{j}$ is adjacent to the already 


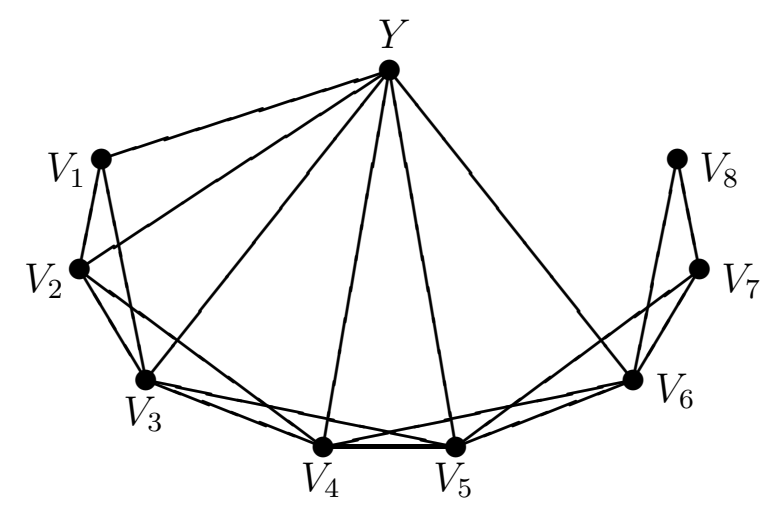

Figure 1: $G_{10: 3}$

identified vertices $V_{j-1}, V_{j-2}, \ldots, V_{j-k+1}$. This uniquely identifies each of these vertices. Finally, for $n-2 k+3 \leq j \leq n-k$, each $V_{j}$ is uniquely identified by its degree in $F$ and its adjacency to $V_{n-k+1}$.

Thus $G$ is 1-distinguishable and by Theorems 2 and 3 we get that $\left(K_{n: k}\right)^{c}$ is 2distinguishable. Thus we may conclude that $K_{n: k}$ is 2-distinguishable.

Case 2) Suppose that $2 k+1 \leq n \leq 3 k-1$. Then $n=2 k+r$ where $1 \leq r \leq k-1$. Note that here we work with $K_{n: k}$ rather than its complement. Let $H$ denote the subgraph of $K_{n: k}$ induced by $\left\{V_{i}: 1 \leq i \leq n\right\}$. It is worth remarking that $H$ is $K_{\frac{n}{k}}$, the circular clique $[12,19]$. It is easy to check that the degree of each vertex in $H$ is $n-2 k+1=r+1$. Since any superset of a determining set is determining, $V(H)$ is a determining set. We then find an asymmetric, and therefore 1-distinguishable, induced subgraph of $K_{n: k}$ containing these vertices. By Theorems 2 and 3 this guarantees that $K_{n: k}$ is 2-distinguishable.

Our goal is to create an induced subgraph of $G$ that consists of $H$ with a path of length 2 attached to $V_{1}$ and a path of length 1 attached to $V_{k+1}$. By doing so we differentiate $V_{1}$ and $V_{k+1}$ and thereby create an asymmetric graph. Let $U_{1}, U_{2}$, and $X$ denote the vertices that correspond with the $k$-subsets of $[n]$ indicated below:

$$
\begin{aligned}
& U_{1} \leftrightarrow\{k+1, k+2, \ldots, 2 k-r, 2 k+1, \ldots, 2 k+r\} ; \\
& U_{2} \leftrightarrow\{2 k-r+2, \ldots, 2 k, r+1, \ldots, k, 1\} ; \text { and } \\
& X \leftrightarrow\{2 k+1, \ldots, 2 k+r, r+1, \ldots, k\} .
\end{aligned}
$$

It is straightforward to check that

1. $U_{1} V_{i} \in E\left(K_{n: k}\right)$ if and only if $i=1$;

2. $U_{2} U_{1} \in E\left(K_{n: k}\right)$ and $U_{2} V_{i} \notin E\left(K_{n: k}\right) \forall i \in[n]$; and

3. $X U_{i} \notin E\left(K_{n: k}\right)$ for $i=1,2$ and $X V_{i} \in E\left(K_{n: k}\right)$ if and only if $i=k+1$.

Let $J=J_{n: k}$ denote the subgraph of $K_{n: k}$ induced by $V_{1}, \ldots, V_{n}, U_{1}, U_{2}$, and $X$. By construction $\operatorname{deg}_{J}\left(V_{1}\right)=\operatorname{deg}_{J}\left(V_{k+1}\right)=r+2 ; \operatorname{deg}_{J}(X)=\operatorname{deg}_{J}\left(U_{2}\right)=1 ; \operatorname{deg}_{J}\left(U_{1}\right)=2$; and 
$\operatorname{deg}_{J}\left(V_{i}\right)=r+1$ when $2 \leq i \leq k$ or $k+2 \leq i \leq n$. Just by looking at the degrees of the vertices of $J$ and the degrees of their neighbors within $J$, it is immediate that every automorphism of $J$ fixes $U_{1}, U_{2}, X, V_{1}$, and $V_{k+1}$. Figure 2 shows $J_{8,3}$.

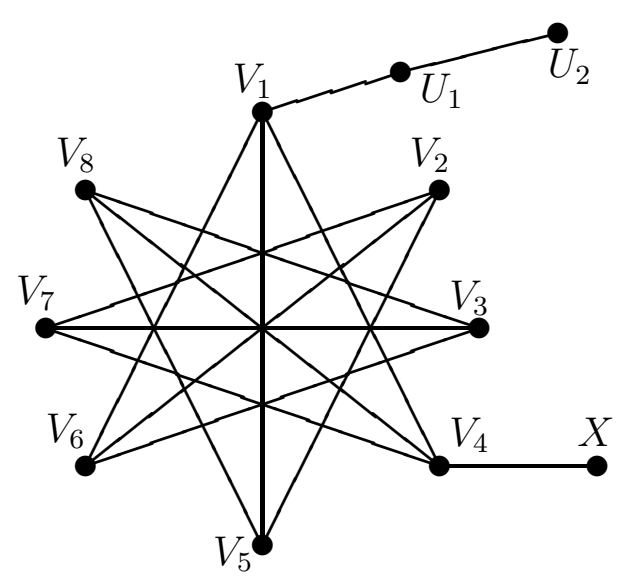

Figure 2: $J_{8,3}$

With reasoning similar to that of the previous case, every automorphism of $J$ that fixes $U_{1}, U_{2}, X, V_{1}$ and $V_{k+1}$ induces an automorphism of $H$ that fixes $V_{1}$ and $V_{k+1}$. Thus, it only remains to show that every automorphism of $H$ that fixes $V_{1}$ and $V_{k+1}$ fixes all of $H$. The following lemma, which may be of independent interest, is useful.

Lemma 1. Suppose $H$ is the subgraph of $K_{n: k}$ induced by $S=\left\{V_{1}, \ldots, V_{n}\right\}$ where $V_{i} \leftrightarrow\{i, \ldots, i+k-1\}$. If $k \geq 3$, then the automorphisms of $H$ that are induced by permutations of $[n]$ preserve or reverse the cyclic ordering of the vertices of $H$.

Proof. If $\sigma \in S_{n}$ is a permutation on [n], let $\tilde{\sigma}$ denote the induced action of $\sigma$ on $V\left(K_{n: k}\right)$ i.e. $\tilde{\sigma}\left(\left\{i_{1}, i_{2}, \ldots i_{k}\right\}\right)=\left\{\sigma\left(i_{1}\right), \sigma\left(i_{2}\right), \ldots, \sigma\left(i_{k}\right)\right\}$. Let $S=\left\{V_{1}, \ldots, V_{n}\right\}$ and suppose $\sigma$ is such that $\tilde{\sigma}(S)=S$. We proceed by contradiction.

Suppose that $\sigma \notin D_{n}$. That is, suppose that $\sigma$ does not preserve the 'cycle modulo $n$.' Then $\sigma$ takes two integers whose difference is 1 , say 1 and 2, and maps them to integers whose difference is more than 1 . Then $\sigma(1)=r \in\{1, \ldots, n\}$ and $\sigma(2) \neq r+1, r-1$.

Since $\sigma(2)$ is not 'next to' $\sigma(1)$, something else that wasn't originally 'next to' 1 is mapped by $\sigma$ to be 'next to' $\sigma(1)$. More precisely, since $\sigma(2)$ is neither $r+1$ nor $r-1$, there exist $t \notin\{1,2, n\}$ so that $\sigma(t)=r+1$ or $\sigma(t)=r-1$. Without loss of generality assume there is such a $t$ for which $\sigma(t)=r+1$.

Since $\tilde{\sigma}\left(V_{1}\right) \in S$, there exists $i$ so that $\tilde{\sigma}\left(V_{1}\right)=\{i, \ldots, i+k-1\}$ when put in proper cyclic order. We can assume that $\sigma(1)<i+k-1$. (If not, replace $\sigma$ by $(12 \cdots n)^{-1} \sigma$ and repeat the argument.) Since $r=\sigma(1)$ is not the last integer when $\{\sigma(1), \ldots, \sigma(k)\}$ is put in cyclic order as $\{i, \ldots, i+k-1\}$, then $r+1=\sigma(t)$ must be in $\{\sigma(1), \ldots, \sigma(k)\}$. 
This implies that $t \in\{1, \ldots, k\}$. Since $t \neq 1,2$, we have that $t \in\{3, \ldots, k\}$. Since $k \geq 3$, such a $t$ can exist.

Now consider $\tilde{\sigma}\left(V_{n-k+3}\right)$. Since $3 \leq t \leq k$ and $n>2 k, t \notin\{n-k+3, \ldots, n, 1,2\}$. Thus $\sigma(1)$ must be the last integer in $\tilde{\sigma}\left(V_{n-k+3}\right) \leftrightarrow\{\sigma(n-k+3), \ldots, \sigma(n), \sigma(1), \sigma(2)\}$ when cyclically ordered. But similarly we also have that $t \notin\{n-k+2, \ldots, n, 1\}$, so $\sigma(1)$ must also be the last integer in the the cyclic ordering of $\tilde{\sigma}\left(V_{n-k+2}\right) \leftrightarrow\{\sigma(n-k+$ $2), \ldots, \sigma(n), \sigma(1)\}$. Since the $V_{i}$ are uniquely determined by their final elements, this implies that $\tilde{\sigma}\left(V_{n-k+3}\right)=\tilde{\sigma}\left(V_{n-k+2}\right)$ which implies that $V_{n-k+3}=V_{n-k+2}$, a contradiction. Thus $\sigma \in D_{n}$.

Notice that when $\sigma \in D_{n}$ is a power of the $n$-cycle (that is, it contains no reflection), then $\tilde{\sigma}\left(V_{i}\right)=V_{\sigma(i)}$. When $\sigma \in D_{n}$ contains a reflection, then $\tilde{\sigma}\left(V_{i}\right)=V_{\sigma(i+k-1)}$. Thus every automorphism in $\operatorname{Aut}(H)$ induced by permutation of $[n]$ preserves or reverses the cyclic ordering of $S=\left\{V_{1}, \cdots, V_{n}\right\}$.

By the preceding lemma, since $V_{1}$ and $V_{k+1}$ are not antipodal on the $n$ cycle, the only automorphism of $H$ that fixes both $V_{1}$ and $V_{k+1}$ is the identity. By the remarks preceding the lemma every automorphism of $J$ must fix $V_{1}$ and $V_{k+1}$. Consequently the only automorphism of $K_{n: k}$ that maps $J$ to $J$ is the identity. Thus $J$ is an asymmetric graph and $V(J)$ is a 1-distinguishable determining set for $K_{n: k}$. It follows that $\operatorname{Dist}\left(K_{n: k}\right)=2$.

Acknowledgment: We are indebted to an anonymous referee for discovering a minor error in an earlier version of this paper.

\section{References}

[1] Michael O. Albertson. Distinguishing Cartesian powers of graphs. Electron. J. Combin., 12:Note 17 (electronic), 2005.

[2] Michael O. Albertson and Debra L. Boutin. Distinguishing geometric graphs. J. Graph Theory, 53:135-150, 2006.

[3] Michael O. Albertson and Karen L. Collins. Symmetry breaking in graphs. Electron. J. Combin., 3(1):Research Paper 18 (electronic), 1996.

[4] Bill Bogstad and Lenore J. Cowen. The distinguishing number of the hypercube. Discrete Math., 283(1-3):29-35, 2004.

[5] Debra L. Boutin. Identifying graph automorphisms using determining sets. Electron. J. Combin., 13(1):Research Paper 78 (electronic), 2006.

[6] Melody Chan. The distinguishing number of the direct product and the wreath product action. J. Algebr. Comb., 24:331-335, 2006.

[7] Melody Chan. The maximum distinguishing number of a group. Electron. J. Combin., 13:Research Paper 70 (electronic), 2006.

[8] Christine T. Cheng. On computing the distinguishing numbers of trees and forests. Electron. J. Combin., 13(1):Research Paper 11 (electronic), 2006. 
[9] Karen L. Collins and Ann N. Trenk. The distinguishing chromatic number. Electron. J. Combin., 13(1):Research Paper 16 (electronic), 2006.

[10] Chris Godsil and Gordon Royle. Algebraic graph theory, volume 207 of Graduate Texts in Mathematics. Springer-Verlag, New York, 2001.

[11] R. L. Graham, M. Grötschel, and L. Lovász, editors. Handbook of combinatorics. Vol. 1, 2. Elsevier Science B.V., Amsterdam, 1995.

[12] Pavol Hell and Jaroslav Nešetřil. Graphs and homomorphisms, volume 28 of Oxford Lecture Series in Mathematics and its Applications. Oxford University Press, Oxford, 2004.

[13] Wilfried Imrich and Sandi Klavžar. Distinguishing Cartesian powers of graphs. $J$. Graph Theory, 53(3):250-260, 2006.

[14] Sandi Klavžar, Tsai-Lien Wong, and Xuding Zhu. Distinguishing labellings of group action on vector spaces and graphs. J. Algebra, 303(2):626-641, 2006.

[15] Sandi Klavzar and Xuding Zhu. Cartesian powers of graphs can be distinguished with two labels. European J. Combinatorics. (to appear).

[16] Frank Rubin. Problem 729: the blind man's keys. J. Recreational Mathematics, 11(2):128, 1979. Solution in 12 (2) (1980).

[17] Thomas Tucker. Distinguishability of maps. preprint (2005).

[18] Julianna Tymoczko. Distinguishing numbers for graphs and groups. Electron. J. Combin., 11(1):Research Paper 63 (electronic), 2004.

[19] Xuding Zhu. Circular chromatic number: a survey. Discrete Math., 229(1-3):371-410, 2001. Combinatorics, graph theory, algorithms and applications.

[20] Xuding Zhu and Tsai-Lien Wong. Distinguishing sets of the actions of symmetric groups. preprint (2005). 